\title{
Expansion of a random field with interval correlation length using interval fields
}

\author{
Wim Verhaeghe ${ }^{*}$ Wim Desmet $\stackrel{\dagger}{\dagger}$ Dirk Vandepitte ${ }^{\dagger}$ and David Moens ${ }^{\ddagger}$
}

\begin{abstract}
This paper discusses the application of interval fields for the analysis of uncertain mechanical structures. More specifically, this work focuses on representing uncertainties with a spatially distributed influence in the context of finite element analysis. First, the concept of interval fields is briefly reviewed. Next, random fields are presented, with a focus on the influence of an uncertain correlation length on its discretization. The methods for applying the interval field framework to represent the uncertain correlation length are explained in the next section. Finally, the application of interval fields for representing a random field expansion in the uncertain correlation length space is illustrated using a numerical example.
\end{abstract}

\section{Introduction}

In recent years many methods, both probabilistic and non-probabilistic, have been developped to deal with scalar parameter uncertainties in Finite Element (FE) analysis. These approaches cover a large part of the uncertainty problems in engineering. Uncertainty and variability with a spatial character (e.g. material properties or load distributions) require a specific approach. The mathematical concept of random fields is well developped for probabilistic methods. However it does not yet have a counterpart in non-probabilistic techniques.

In both frameworks (probabilistic and non-probabilistic) two extreme methods of handling such uncertainties are often used. The first method consists in the application of a single interval or random variable to represent a parameter for the entire model. As such the uncertain parameter is considered constant within one realization of the model. The second method consists in the introduction of an interval or random variable for every individual element in the FE model. In this way the uncertainty is represented as if it is spatially independent. Both extremes are clearly unrealistic. The first by assuming total dependency, the second by ignoring all possible dependency.

The solution to this fundamental problem can be found in presenting such an uncertainty in a framework that allows for the representation of the uncertainty and the spatial dependency in clearly separated entities. The spatial dependency is never completely uncertain, nor is the uncertainty completely spatially dependent. This insight should enable us to represent both aspects separately.

The authors have developped an interval field framework. ${ }^{1}$ In this framework an explicit and implicit description are developped. In the explicit interval field a set of constant base vectors describes the spatial dependency. Every vector of the set has an associated interval factor, which describes the uncertainty. This interval factor scales the associated base vector. In this way the uncertainty space is drastically reduced from independent uncertainty about every component of the field, into uncertainty about the value of a limited number of well chosen interval factors. In the implicit interval field a single vector describes the multiple dependent uncertainties by having implicit functions for each of its components. These implicit functions all depend on the same limited number of well chosen interval factors. This framework is further introduced in section II.

The choice of the base vectors by the analysist/expert is at the same time an advantage and a shortcoming of

${ }^{*} \mathrm{PhD}$ student, K.U.Leuven, Dept. of Mechanical Engineering, Celestijnenlaan 300B, 3001 Heverlee, Belgium, wim.verhaeghe@mech.kuleuven.be

${ }^{\dagger}$ Professor, K.U.Leuven, Dept. of Mechanical Engineering, Celestijnenlaan 300B, 3001 Heverlee, Belgium

${ }^{\ddagger}$ Professor, K.U.Leuven Association, Lessius Hogeschool - Campus De Nayer, Dept. of Applied Engineering, J. De Nayerlaan 5, 2860 Sint-Katelijne-Waver, Belgium, david.moens@mech.kuleuven.be 
the interval field framework when representing spatially dependent input uncertainty. This clearly subjective choice acknowledges the spatial dependency but might not take it into account in a mathematically correct manner.

In the probabilistic framework the concept of random fields ${ }^{2}$ has gained particular attention as an intermediate solution in between the two extremes described above. The crucial choice to be made in its application is the autocorrelation structure of such a field, since its autocorrelation structure, in particular its correlation length, determines completely its discretization into a set of uncorrelated random variables and corresponding deterministic functions (its base vectors). Very often the correlation length of such a random field is unknown but chosen to be constant. The lack of knowledge on the correlation length is often covered with a sensitivity analysis of the final result on different values of the correlation length. In the authors' opinion, the correlation length itself is an uncertain parameter, which affects the uncertainty in the final $\mathrm{FE}$ results.

In confronting the mathematically well developped discretization of a random field with the uncertain correlation length it appears too little uncertainty is left in the actual problem when the correlation length is taken to be constant. By acknowledging the subjective choice of base vectors in the interval field framework it may appear that this puts too much uncertainty into the actual problem. By combining the advantages of both methods this paper presents an interval field method that attempts to capture the influence of an interval correlation length on a random field representation of an uncertain FE input.

After a short introduction to interval fields in section II, section III describes the necessary concepts of the random field framework. It is shown how the interval field and random field concept can be integrated to deal with random fields having an interval correlation length in section IV. Finally section V discusses the application of the developed method to both an academic and an industrially relevant FE model.

\section{Interval fields}

The interval field technique has two general ways of solving the dependency problem: explicit interval fields and implicit interval fields. ${ }^{1}$

\section{II.A. Explicit interval field}

To describe a spatially dependent variation, numerical modelling approaches often use some type of shape functions (e.g. the modes used to represent the dynamic behaviour of a structure using the modal superposition technique). The actual solution is a linear combination of these shape functions.

Accordingly, the explicit interval field $\mathbf{x}^{F}$ is defined as a superposition of $n_{b}$ base vectors $\boldsymbol{\psi}_{i}$ using interval factors $\alpha_{i}^{I}$ :

$$
\mathbf{x}^{F}=\sum_{i=1}^{n_{b}} \alpha_{x, i}^{I} \boldsymbol{\psi}_{x, i}
$$

The base vectors represent a limited set of reference patterns, each of which is scaled by an interval factor. The components of the interval fields themselves are coupled through the reference patterns. Once the reference patterns are chosen, the definition of the interval field requires the specification of the interval factors that define the field on $x$, which can be assembled in a classical (hypercubic) interval vector $\boldsymbol{\alpha}_{x}^{I}$. In matrix notation, the interval field is denoted as:

$$
\mathbf{x}^{F}=\left[\boldsymbol{\psi}_{x}\right] \boldsymbol{\alpha}_{x}^{I}
$$

The application of explicit interval fields on the input side of an analysis is rather straightforward. Since expert knowledge about the modelled system dominates the definition of the uncertainties, the freedom in choosing the base vectors is ideal to reflect this knowledge (for example: the sinusodal (= base vector) deviation of the thickness of a rolled plate with uncertain amplitude (= interval factor)). On the other hand, as mentioned in section I, the spatial dependency is clearly subjectively chosen and might benefit from a more rigorous approach. The main limitation of the explicit interval field is that its definition only allows a linear relation between the base vectors and the interval factors. Furthermore, in order to obtain an explicit interval field that introduces no conservatism in its derived response variables, these parameters should be 
completely independent.

\section{II.B. Implicit interval field}

The implicit interval field is an alternative mathematical formalism to express dependency between uncertain vector components. It is based on implicit functions $\phi_{y, i}\left(\boldsymbol{\alpha}_{y}\right)$ of a set of predefined interval factors $\boldsymbol{\alpha}_{y}$, describing the range of each individual component of the vector:

$$
\left.\mathbf{y}^{F}=\left\{\begin{array}{c}
\phi_{y, 1}\left(\boldsymbol{\alpha}_{y}\right) \\
\vdots \\
\phi_{y, n}\left(\boldsymbol{\alpha}_{y}\right)
\end{array}\right\}, \boldsymbol{\alpha}_{y} \in \boldsymbol{\alpha}_{y}^{I}\right\}=\left\{\phi_{y}\left(\boldsymbol{\alpha}_{y}\right), \boldsymbol{\alpha}_{y} \in \boldsymbol{\alpha}_{y}^{I}\right\}
$$

By introducing appropriate functions $\phi_{i}\left(\boldsymbol{\alpha}_{y}\right)$ for all components of the vector, the coupling between its components is incorporated implicitly through the interval factors.

The main advantage of this approach is that it no longer assumes a linear relationship between the interval factors and the field. Furthermore, the set of interval factors is chosen first, after which the implicit relationship with the vector components is determined through the numerical analysis. As such, a set of independent interval factors can always be guaranteed. In many cases, the actual set of interval uncertainites present at the input side of the problem is a very good candidate set, as they often are known (or considered) to be independent. The implicit interval field is especially suited for application at the output side of an analysis. Based on a few exact evaluations in the uncertainty space, a response surface can be built for every component of an output vector. These response surfaces may represent the implicit functions $\phi_{i}\left(\boldsymbol{\alpha}_{y}\right)$ described above.

\section{Random fields}

The objective of a random field is to represent a spatial variation of a specific model property by a stochastic variable defined over the region on which the variation occurs. ${ }^{2}$ A random field can thus be denoted as $H(\mathbf{x}, \theta)$ with $\mathbf{x}$ the spatial coordinate and $\theta$ the outcome of a random phenomenon. A random field is a random variable for a given $\mathbf{x}_{\mathbf{0}}$ and is a realization of the field for a given $\theta_{0}$. The specification of a random field generally comes down to the specification of the spatial evolution of the first two statistical moments of the field variable and a corresponding covariance function, expressing the spatial dependency of the field variable. In most cases (also in this paper) the random field is considered to be weakly stationary, resulting in a constant for the first few statistical moments throughout the spatial domain (zero mean and unit variance in this paper). Furthermore the covariance function for weakly stationary random fields depends only on the distance between observation points, not on their actual location.

The application of the concept of random fields in a numerical modelling framework requires some sort of discretisation of the spatially varying stochastic field over the defined geometry. A good overview of methods can be found in the report by Sudret and Der Kiureghian. ${ }^{3}$ The technique studied here is the KarhunenLoève expansion ${ }^{4}$ that has gained particular attention in literature. This approach is based on the spectral decomposition of the autocovariance function $C_{H H}\left(\mathbf{x}_{1}, \mathbf{x}_{2}\right)$. The set of deterministic functions over which any realization of the field $H\left(\mathbf{x}, \theta_{0}\right)$ is expanded is defined by the eigenvalue problem:

$$
\int_{\Omega} C_{H H}\left(\mathbf{x}_{\mathbf{1}}, \mathbf{x}_{\mathbf{2}}\right) \varphi_{i}\left(\mathbf{x}_{\mathbf{2}}\right) d \Omega_{\mathbf{x}_{\mathbf{2}}}=\lambda_{i} \varphi_{i}(\mathbf{x})
$$

with $\Omega$ the spatial domain and $i=1, \ldots$. Analytical solutions exist only for a limited number of autocovariance functions, but a Galerkin-type procedure is documented to find the eigenfunctions approximately. ${ }^{4}$ Once the eigenfunctions are found the random field can be expressed as:

$$
H(\mathbf{x}, \theta)=\sum_{i=1}^{\infty} \sqrt{\lambda_{i}} \xi_{i}(\theta) \varphi_{i}(\mathbf{x})
$$

with $\left\{\xi_{i}\right\}$ a set of orthonormal random variables. This expansion is truncated after $\mathrm{N}$ terms to reduce the computational costs of the subsequent stochastic analysis. 


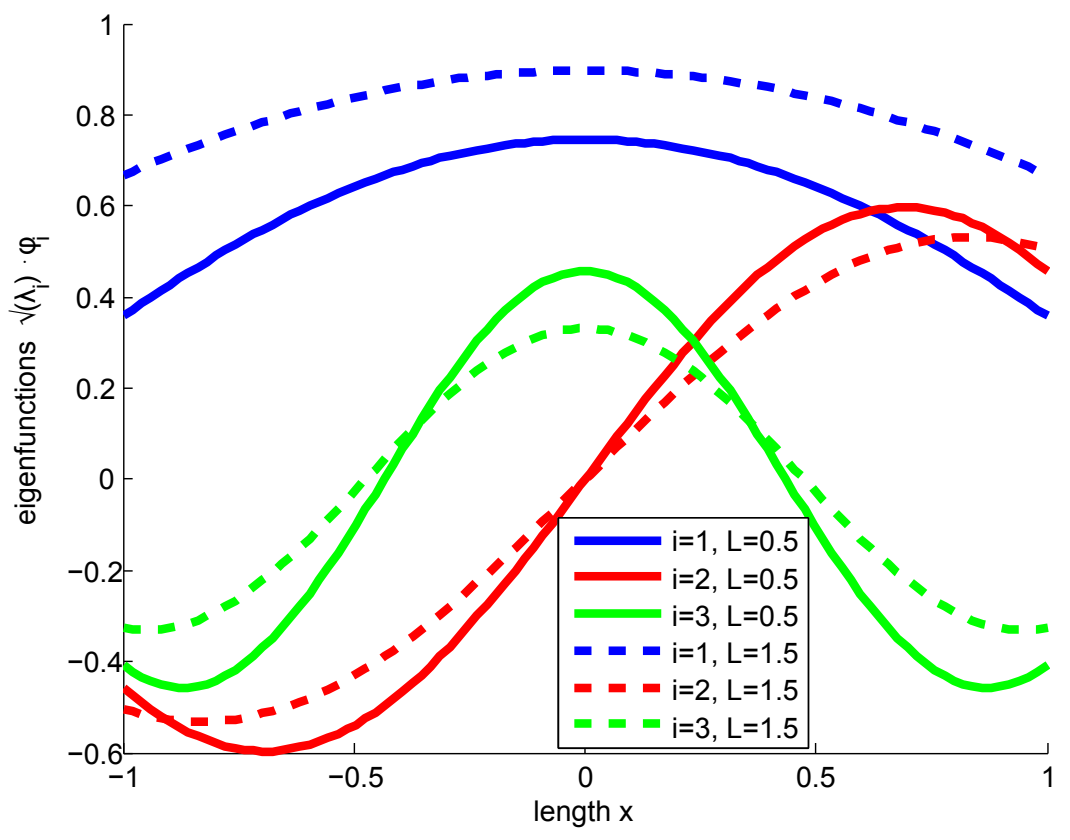

Figure 1. Expansion of random field for correlation length $L=0.5$ and 1.5

In this paper an exponential autocovariance function in one dimension is assumed:

$$
C_{H H}\left(x_{1}, x_{2}\right)=e^{-\frac{\left|x_{1}-x_{2}\right|}{L}}
$$

with $L$ the correlation length. The influence of an uncertain correlation length on the expansion of this random field is illustrated in figure 1 . The field is defined over $x \in\left[\begin{array}{ll}-1 & 1\end{array}\right]$ and the first three eigenfunctions of the expansion are shown for two different values of the correlation length. In the remainder of this paper the autocovariance function is denoted by $C_{H H}\left(x_{1}, x_{2}, L\right)$ to stress its dependence on the correlation length $L$.

\section{Interval fields to represent random fields with interval correlation length}

This section presents the application of interval fields to represent a random field with interval correlation length. The goal is to efficiently represent a random field with interval correlation length. Such an efficient representation can be used in an optimisation and anti-optimisation approach over the uncertainty space (which includes the uncertain correlation length) to find the bounds on a requested output.

The general concept is to use the eigenfunctions of the random field's expansion as the base vectors of the interval field description. In both the explicit and implicit interval field description the random field is expanded for a limited number of correlation length values. Based on these expansions the interval field description represents the random field for all the possible correlation lengths by means of a response surface methodology.

Let the correlation length $L \in\left[\begin{array}{ll}L_{\min } & L_{\max }\end{array}\right]$ and $L_{n o m}=\frac{L_{\min }+L_{\max }}{2}$, then the expansion of the random field for $L_{\text {min }}, L_{n o m}$ and $L_{\max }$ results in:

$$
\left[\left\{\varphi_{i}^{\min }(\mathbf{x})\right\}\left\{\varphi_{i}^{\text {nom }}(\mathbf{x})\right\}\left\{\varphi_{i}^{\max }(\mathbf{x})\right\}\right]
$$

The use of these eigenfunctions is different for the explicit and implicit version of the interval fields.

\section{IV.A. Explicit interval field}

The eigenfunctions from equation (7) are considered as the base vectors $\left[\boldsymbol{\psi}_{x}\right]$ from equation (2) after orthonormalization (denoted by $\bar{\varphi}$ ). To determine a value for the interval factors $\boldsymbol{\alpha}_{x}^{I}$ given a correlation length $L^{*} \in\left[L_{\min } L_{\max }\right]$ the following procedure is proposed. The autocovariance matrix is built for the considered 
correlation length. The base vectors, grouped by equal $i$, are used to project this autocovariance matrix for every $i$ :

$$
C_{H H}^{r e d}\left(x_{1}, x_{2}, L^{*}\right)=\left[\bar{\varphi}_{i}^{\min }(\mathbf{x}) \bar{\varphi}_{i}^{n o m}(\mathbf{x}) \bar{\varphi}_{i}^{\max }(\mathbf{x})\right]^{T} C_{H H}\left(x_{1}, x_{2}, L^{*}\right)\left[\bar{\varphi}_{i}^{\min }(\mathbf{x}) \bar{\varphi}_{i}^{n o m}(\mathbf{x}) \bar{\varphi}_{i}^{\max }(\mathbf{x})\right]
$$

From the spectral decomposition of $C_{H H}^{r e d}\left(x_{1}, x_{2}, L^{*}\right)$ the contributions of (i.e. the value of the interval factor for) the different base vectors can be derived:

$$
\left(\sqrt{\lambda_{i}} \varphi_{i}(\mathbf{x})\right)_{L^{*}} \approx\left[\bar{\varphi}_{i}^{\min }(\mathbf{x}) \bar{\varphi}_{i}^{n o m}(\mathbf{x}) \bar{\varphi}_{i}^{\max }(\mathbf{x})\right]([\chi][\sqrt{\kappa}] \boldsymbol{\beta})_{L^{*}}
$$

with $[\chi]$ the $(3 \times 3)$ matrix containing the eigenvectors and $[\sqrt{\kappa}]$ the diagonal matrix containing the square roots of the eigenvalues of $C_{H H}^{r e d}\left(x_{1}, x_{2}, L^{*}\right)$. The projection (8) might result in a different order of the eigenvectors of $C_{H H}^{r e d}\left(x_{1}, x_{2}, L^{*}\right)$ compared to the order of the eigenvectors of $C_{H H}\left(x_{1}, x_{2}, L^{*}\right)$. $\boldsymbol{\beta}$ is a column vector having a unit value for its component that selects the correct eigenvector appropriately based on the similarity of the corresponding eigenvalues. The product of $([\chi][\sqrt{\kappa}] \boldsymbol{\beta})_{L^{*}}$ forms a vector representing a value of the interval factors $\boldsymbol{\alpha}_{x}^{I}$ of equation (2).

The serious reduction of the eigenvalue problem resulting from the projection of $C_{H H}\left(x_{1}, x_{2}, L^{*}\right)$ on a limited number of base vectors allows for the fast calculation of approximating eigenvectors of the autocovariance matrix. The fast calculation is a first step in the efficient representation of the random field in the uncertain correlation length space. Using this representation an (anti-)optimisation in this space becomes feasible.

To avoid building the autocovariance matrix for every correlation length $L^{*} \in\left[L_{\min } L_{\max }\right]$, then projecting it on the base vectors and solving the reduced eigenvalue problem to find values of the interval factors, one can perform this procedure only for a limited number of correlation lengths. Based on this limited number of solutions, a response surface can be built for the interval factors in the correlation length space. See section $\mathrm{V}$ for an example of this approach. This approach results in an even faster calculation of the approximating eigenvectors.

\section{IV.B. Implicit interval field}

In the implicit variant of the interval field functions are constructed for every component of the field. The eigenfunctions from equation (7) together with their scaling eigenvalues

$$
\left[\left\{\sqrt{\lambda_{i}^{\min }} \varphi_{i}^{\min }(\mathbf{x})\right\}\left\{\sqrt{\lambda_{i}^{\text {nom }}} \varphi_{i}^{\text {nom }}(\mathbf{x})\right\}\left\{\sqrt{\lambda_{i}^{\max }} \varphi_{i}^{\max }(\mathbf{x})\right\}\right]
$$

are used to build a response surface in the correlation length space. Given these three response points a quadratic function for every component can be constructed:

$$
\left\{\sqrt{\lambda_{i}} \varphi_{i}(\mathbf{x})\right\}_{L^{*}} \approx\left\{\sqrt{\lambda_{i}^{n o m}} \varphi_{i}^{\text {nom }}(\mathbf{x})\right\}+\left\{\sqrt{\lambda_{i}^{\text {linear }}} \varphi_{i}^{\text {linear }}(\mathbf{x})\right\} * \Delta L^{*}+\left\{\sqrt{\lambda_{i}^{\text {quadratic }}} \varphi_{i}^{\text {quadratic }}(\mathbf{x})\right\} * \Delta\left(L^{*}\right)^{2}
$$

with $\Delta L^{*}=\frac{L^{*}-L_{n o m}}{L_{n o m}}$ the relative deviation of the correlation length from its nominal value. Other types of response surfaces are also possible, see e.g. section $\mathrm{V}$ for an exponential response surface.

\section{Numerical application}

The interval field, both its explicit and implicit variant, is used to describe the expansion of a random field with interval correlation length. The accuracy of its approximation of the eigenfunctions is checked with respect to the correct expansion for a given correlation length.

A $1 D$ spatial domain with $x \in[-11]$ and an autocovariance function of the exponential form as in

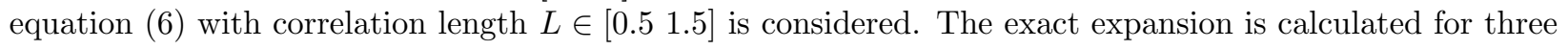
samples: $L_{\min }=0.5, L_{\text {nom }}=1$ and $L_{\max }=1.5$.

For the implicit interval field a quadratic, as in equation (11), and mixed linear/exponential

$$
\left\{\sqrt{\lambda_{i}} \varphi_{i}(\mathbf{x})\right\}_{L^{*}} \approx\left\{\sqrt{\lambda_{i}^{c s t}} \varphi_{i}^{c s t}(\mathbf{x})\right\}+\left\{\sqrt{\lambda_{i}^{\text {linear }}} \varphi_{i}^{\text {linear }}(\mathbf{x})\right\} * L^{*}+\left\{\sqrt{\lambda_{i}^{\text {exp }}} \varphi_{i}^{\text {exp }}(\mathbf{x})\right\} * e^{\frac{-1}{L^{*}}}
$$



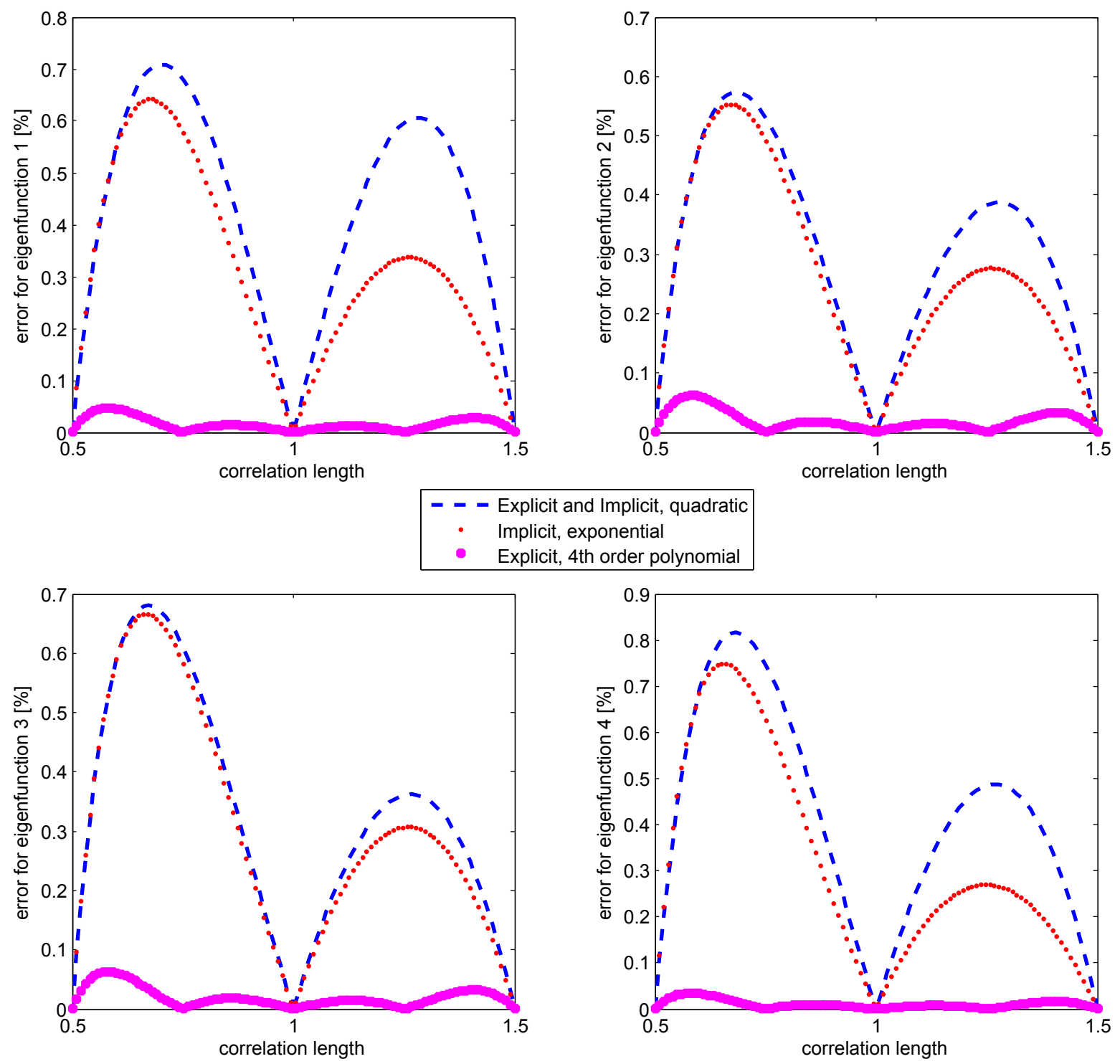

Figure 2. Error of the explicit and implicit interval field representation for the first four eigenfunctions in the correlation length space

response surface is made for the components of the eigenfunction.

For the explicit interval field the reduced eigenvalue problem is solved for three and five values of the correlation length $L$. The three values are the ones for which the exact evaluation was performed, the additional two values are $L=L_{\min }+\frac{L_{n o m}-L_{\min }}{2}$ and $L=L_{\max }-\frac{L_{\max }-L_{n o m}}{2}$.

The relative error is calculated using

$$
\operatorname{error}_{i}=\frac{\sum_{x=-1}^{1}\left|\varphi_{i}(x)-\tilde{\varphi}_{i}(x)\right|}{n_{x} \varphi_{i}(x)_{\max }}
$$

with $n_{x}=100$ the number of samples in the $x$ space and $\varphi_{i}(x)_{\max }$ the maximal value over the space $x$ for the considered eigenfunction. Figure 2 summarizes the errors of the different approximations for the first four eigenfunctions in the random field expansion. The quadratic explicit and implicit interval field (these are inherently the same) perform well (relative error below 1\%), the exponential implicit interval field performs slightly better. Performing two additional projections for the explicit interval field drastically increases the accuracy with only a very small calculation cost (i.e. building the autocovariance matrix, projecting it and solving the reduced eigenvalue problem).

The paper further discusses the application of the presented methodology to a large industrially relevant 


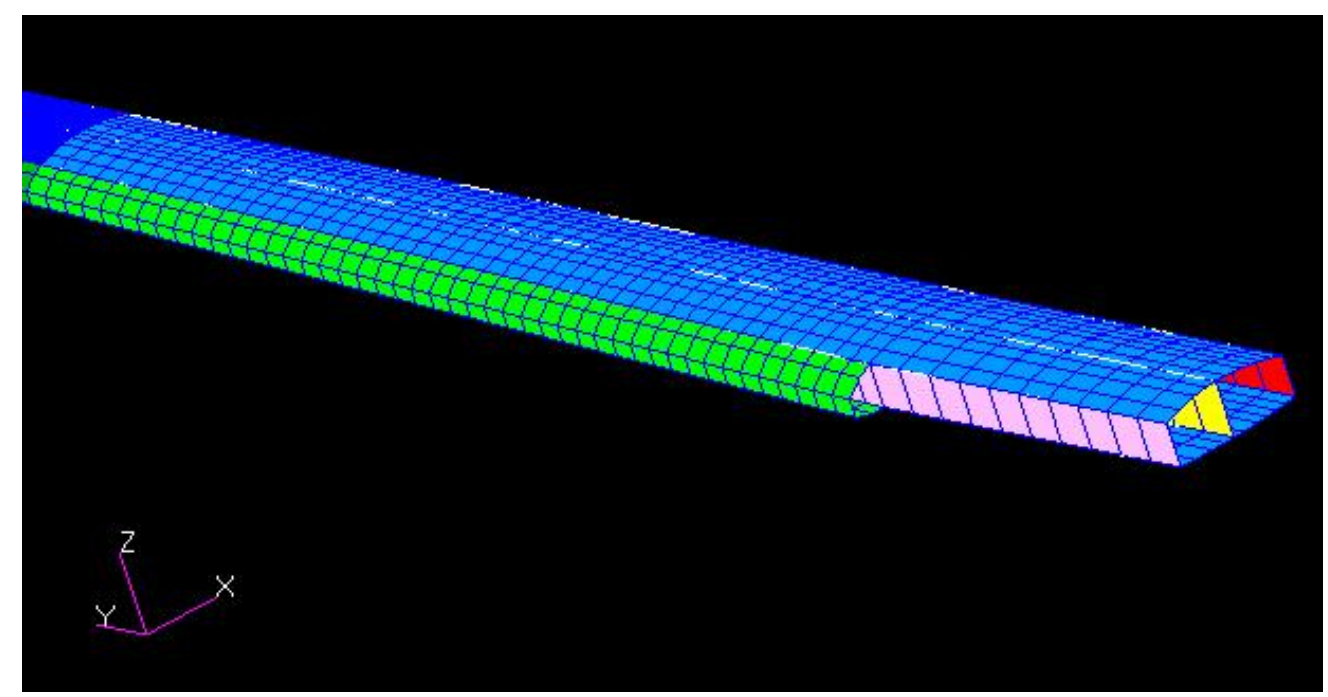

Figure 3. FE model of a composite wing

FE problem of a composite wing (see figure 3). The random field describing the uncertain angle of the fibres in the lay-up is expanded using the presented methodology.

\section{References}

${ }^{1}$ Moens, D., De Munck, M., Desmet, W., and Vandepitte, D., "Numerical dynamic analysis of uncertain mechanical structures based on interval fields," IUTAM Symposium on Vibration Analysis of Structures with Uncertainties, Saint Petersburg, 2009.

${ }^{2}$ Vanmarcke, E., Random fields: analysis and synthesis, MIT Press, Cambridge, 1993.

${ }^{3}$ Sudret, B. and Der Kiureghian, A., "Stochastic Finite Element Methods and Reliability: A State-of-the-Art Report," Department of Civil \&5 Environmental Engineering, University of California, Berkley, Institute of Structural Engineering, Mechanics and Materials, Vol. Tech. Rep., 2000, pp. Report No. UCB/SEMM-2000/08.

${ }^{4}$ Ghanem, R. and Spanos, P., Stochastic finite elements: a spectral approach, Springer-Verlag, New-York, 1991. 